\title{
Volatile Rhenium (I) Compounds with Re-N Bonds and Their Conversion into Oriented Rhenium Nitride Films by Magnetic Field-Assisted Vapor Phase Deposition
}

Michael Frank ${ }^{1}$, Lasse Jürgensen ${ }^{1}$, Jennifer Leduc ${ }^{1}$, Daniel Stadler ${ }^{1}$, David Graf ${ }^{1}$, Isabel Gessner$^{1}$, Fabian Zajusch ${ }^{1}$, Thomas Fischer ${ }^{1}$, Marc-André Rose ${ }^{2}$, David N. Mueller ${ }^{3}$ and Sanjay Mathur ${ }^{1, *}$

${ }^{1}$ Department of Chemistry, Institute of Inorganic Chemistry, University of Cologne, Greinstr. 6, 50939 Cologne, Germany

${ }^{2}$ Institute for Electronic Materials (IWE2), RWTH Aachen University, Sommerfeldstr. 18/24, 52074 Aachen, Germany

${ }^{3}$ Peter Gruenberg Institute, Forschungszentrum Juelich, Wilhelm-Johnen-Str., 52425 Juelich, Germany

*Author to whom correspondence should be addressed; E-Mail: sanjay.mathur@uni-koeln.de Tel.: +49221470 5627; Fax: +492214704899.

Figure S1: Molecular structure of Htfb-mpa (left) and Htfb-dmeda (right). Thermal ellipsoids

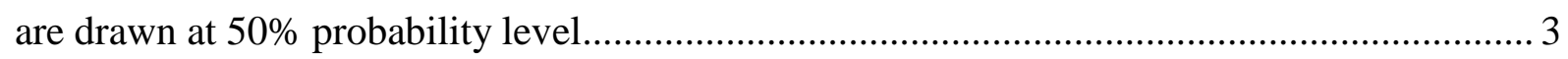

Figure S2: ${ }^{13} \mathrm{C}$ NMR spectrum of $\left[\mathrm{Re}(\mathrm{CO})_{5} \mathrm{Br}\right]$, measured in $\mathrm{CDCl}_{3}$ at room temperature....... 3

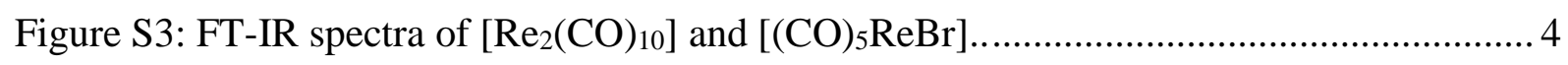

Figure S4: ${ }^{1} \mathrm{H}$ NMR spectrum of 1 , measured in $\mathrm{CDCl}_{3}$ at room temperature........................ 4

Figure S5: ${ }^{19} \mathrm{~F}$ NMR spectrum of $\mathbf{1}$, measured in $\mathrm{CDCl}_{3}$ at room temperature......................... 5

Figure S6: ${ }^{13} \mathrm{C}$ NMR spectrum of $\mathbf{1}$, measured in $\mathrm{CDCl}_{3}$ at room temperature. ...................... 5

Figure S7: Schematic illustration of endo and exo protons in the saturated part of the tridentate ligand and the influence of their chemical shift in the NMR .............................................. 6

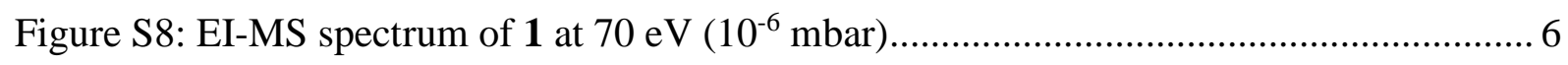

Figure S9: ${ }^{1} \mathrm{H}$ NMR spectrum of 2, measured in $\mathrm{CDCl}_{3}$ at room temperature ......................... 7

Figure S10: ${ }^{19} \mathrm{~F}$ NMR spectrum of 2, measured in $\mathrm{CDCl}_{3}$ at room temperature....................... 7

Figure S11: ${ }^{13} \mathrm{C}$ NMR spectrum of 2, measured in $\mathrm{CDCl}_{3}$ at room temperature ..................... 8

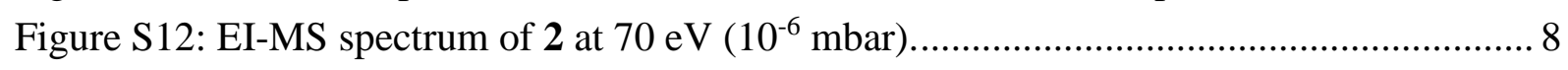

Figure S13: ${ }^{1} \mathrm{H}$ NMR spectrum of $\mathbf{3}$, measured in $\mathrm{CDCl}_{3}$ at room temperature....................... 9

Figure S14: EI-MS spectrum of 3 at $70 \mathrm{eV}\left(10^{-6} \mathrm{mbar}\right)$.................................................... 9

Figure S15: TG curves of $\mathbf{1 - 3}$, measured with a heating rate of $10{ }^{\circ} \mathrm{C} / \mathrm{min}$ under nitrogen .... 10

Figure S16: Molecular structure of 2. Thermal ellipsoids are drawn at 50\% probability level.

Figure S17: FT-IR spectra of 1-3 
Table S1: Crystal X-ray refinement data of Htfb-dmeda, Htfb-mpa, [(CO) $\left.{ }_{3} \operatorname{Re}(\mathrm{tfb}-\mathrm{dmpda})\right]$ and $\left[(\mathrm{CO})_{3} \operatorname{Re}(\mathrm{tfb}-\mathrm{dmeda})\right]$

Figure S18: XPS survey spectrum (left) and high resolution O 1s spectrum (right) of the Rebased film deposited by MO-CVD at $600{ }^{\circ} \mathrm{C}$ on $\mathrm{Si}$.

Figure S19: XPS survey spectrum (left), high resolution O 1s spectrum (middle) and high resolution Re $4 \mathrm{f}$ spectrum (right) of the Re-based film deposited by $\mathrm{mfCVD}$ at $600{ }^{\circ} \mathrm{C}$ on $\mathrm{Si}$.

Table S2: Elemental composition of the deposited films by MO-CVD and mfCVD on Si at $600{ }^{\circ} \mathrm{C}$ analyzed with XPS measurements

Figure S20: Top-view SEM images: Homogenous distributed ReN nanoparticles deposited via CVD on alumina substrate 13

Figure S21: SAED of as deposited ReN (camera length: $600 \mathrm{~mm}$ ) transferred on $\mathrm{Cu}$ grids, indicating the [111], [222] and [422] planes (ICSD: 181297) of polycrystalline deposits. The preferred orientation visible on Si-substrates is lost due to the mechanical transfer to TEM sample holders 13 


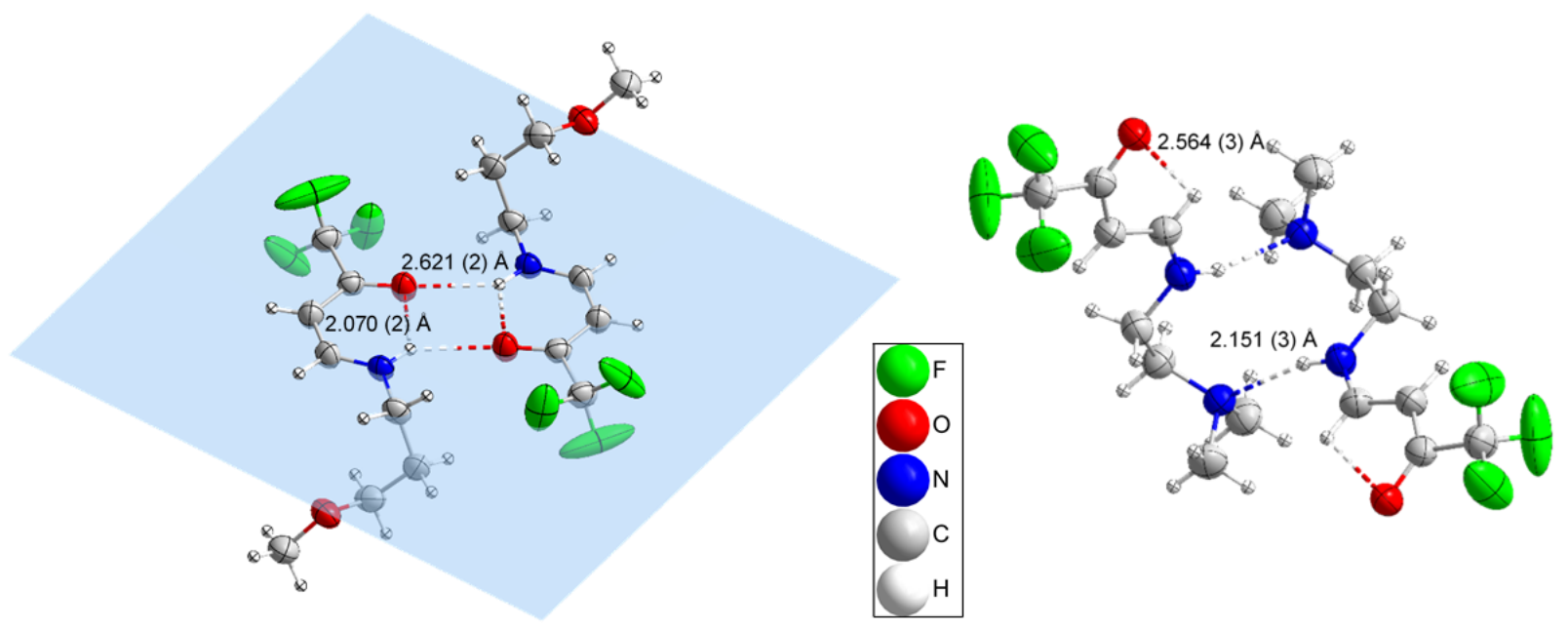

Figure S1: Molecular structure of Htfb-mpa (left) and Htfb-dmeda (right). Thermal ellipsoids are drawn at $50 \%$ probability level.

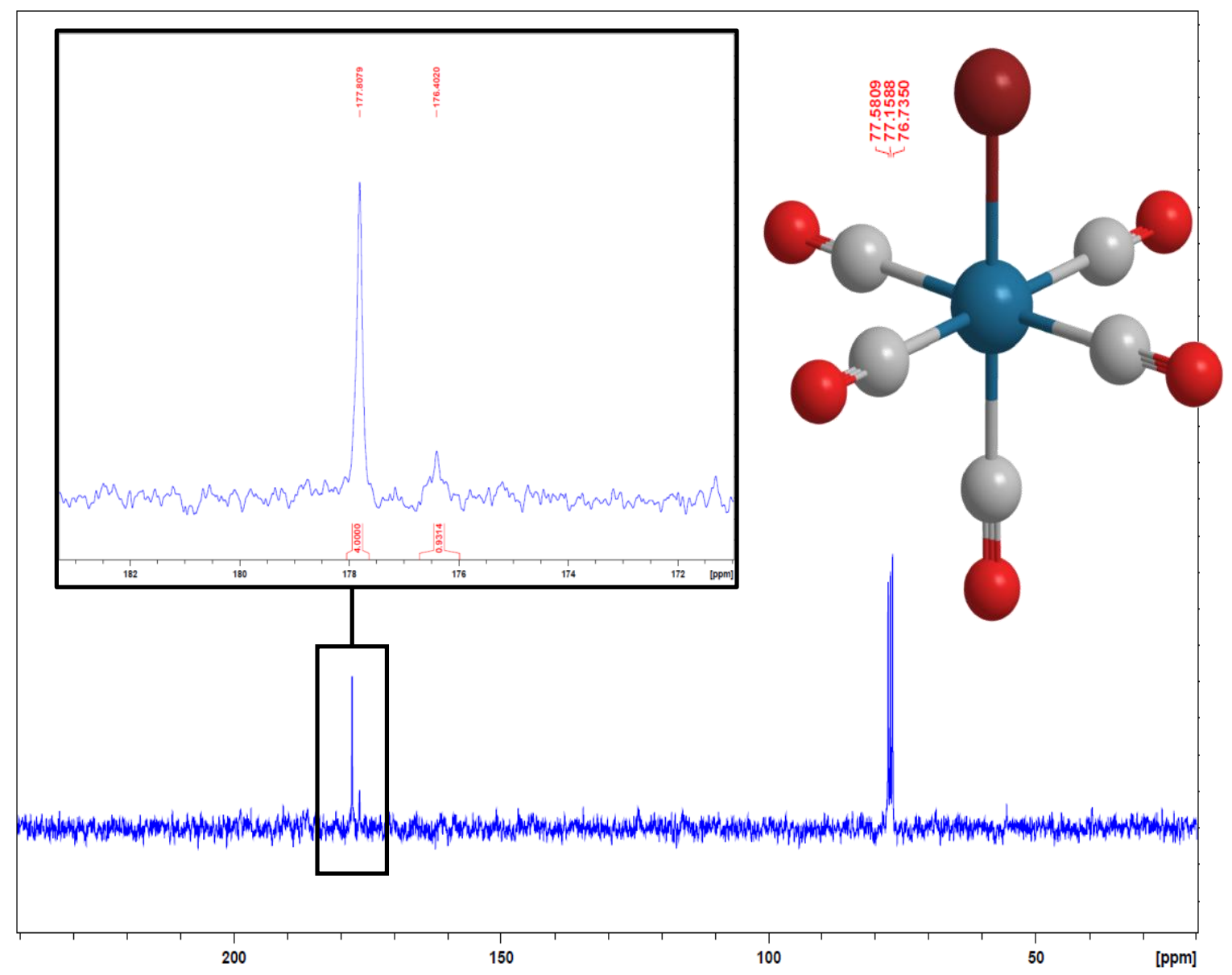

Figure S2: ${ }^{13} \mathrm{C}$ NMR spectrum of $\left[(\mathrm{CO})_{5} \mathrm{ReBr}\right]$, measured in $\mathrm{CDCl}_{3}$ at room temperature. 


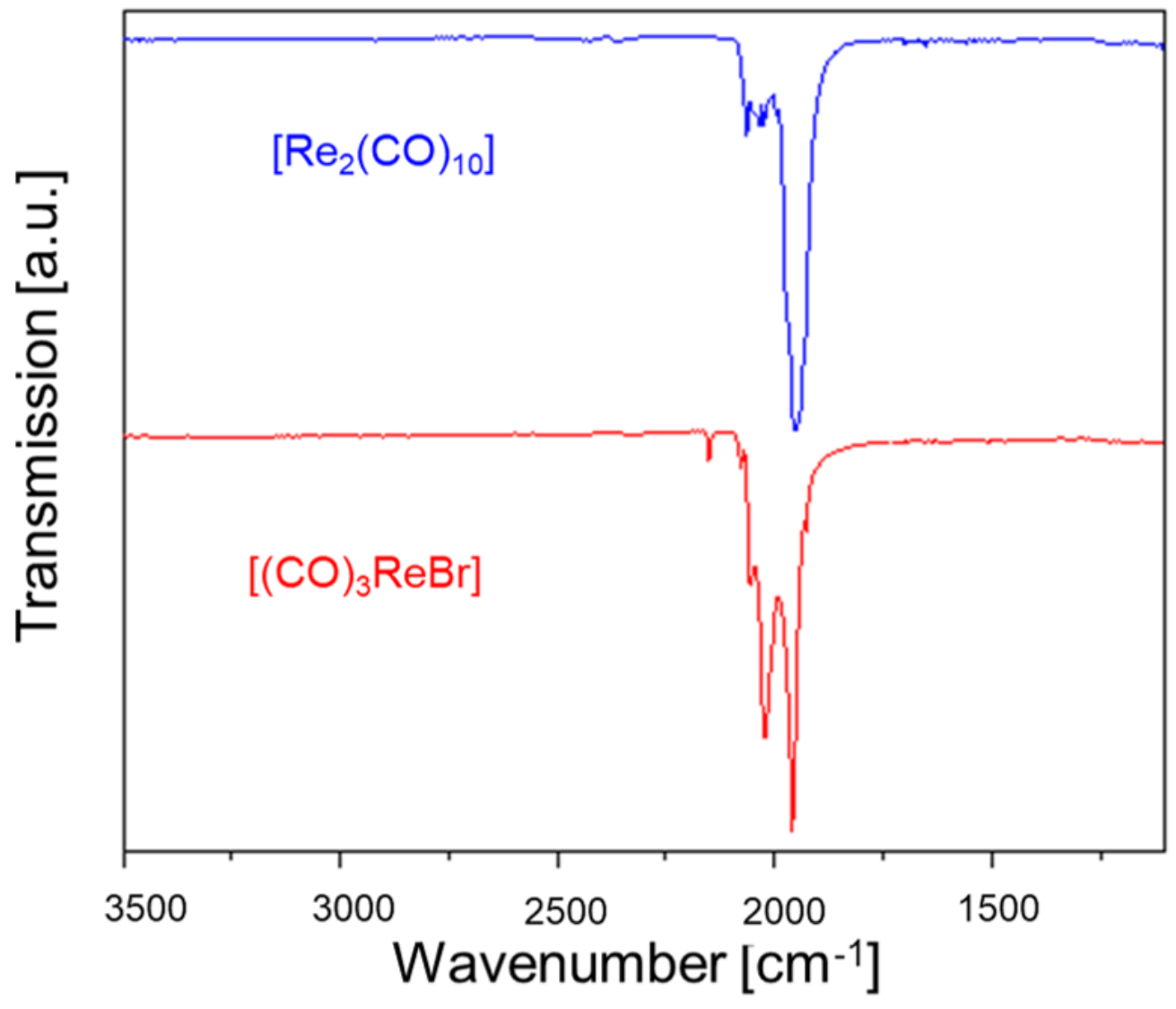

Figure S3: FT-IR spectra of $\left[\mathrm{Re}_{2}(\mathrm{CO})_{10}\right]$ and $\left[(\mathrm{CO})_{5} \mathrm{ReBr}\right]$.

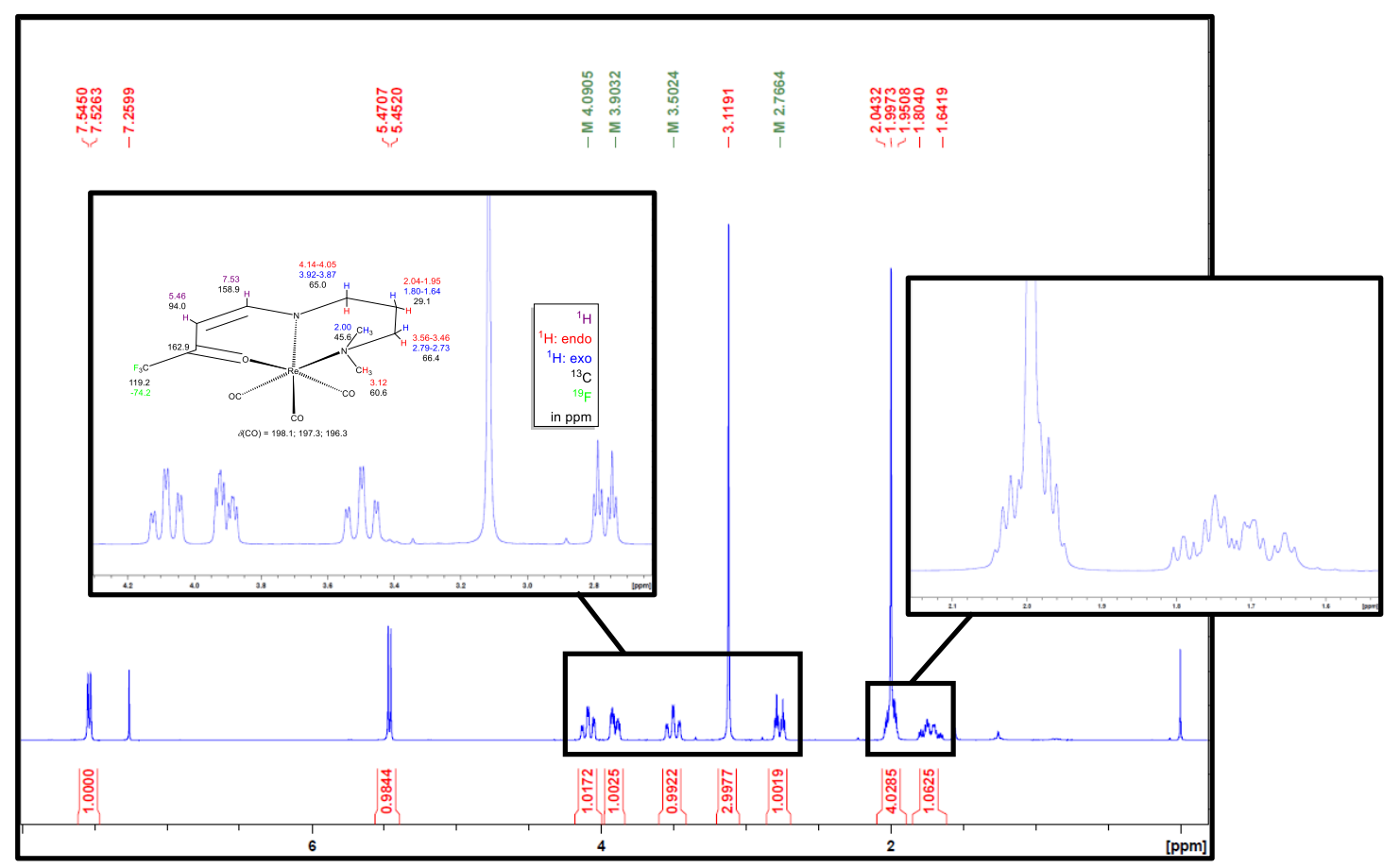

Figure S4: ${ }^{1} \mathrm{H}$ NMR spectrum of $\mathbf{1}$, measured in $\mathrm{CDCl}_{3}$ at room temperature. 


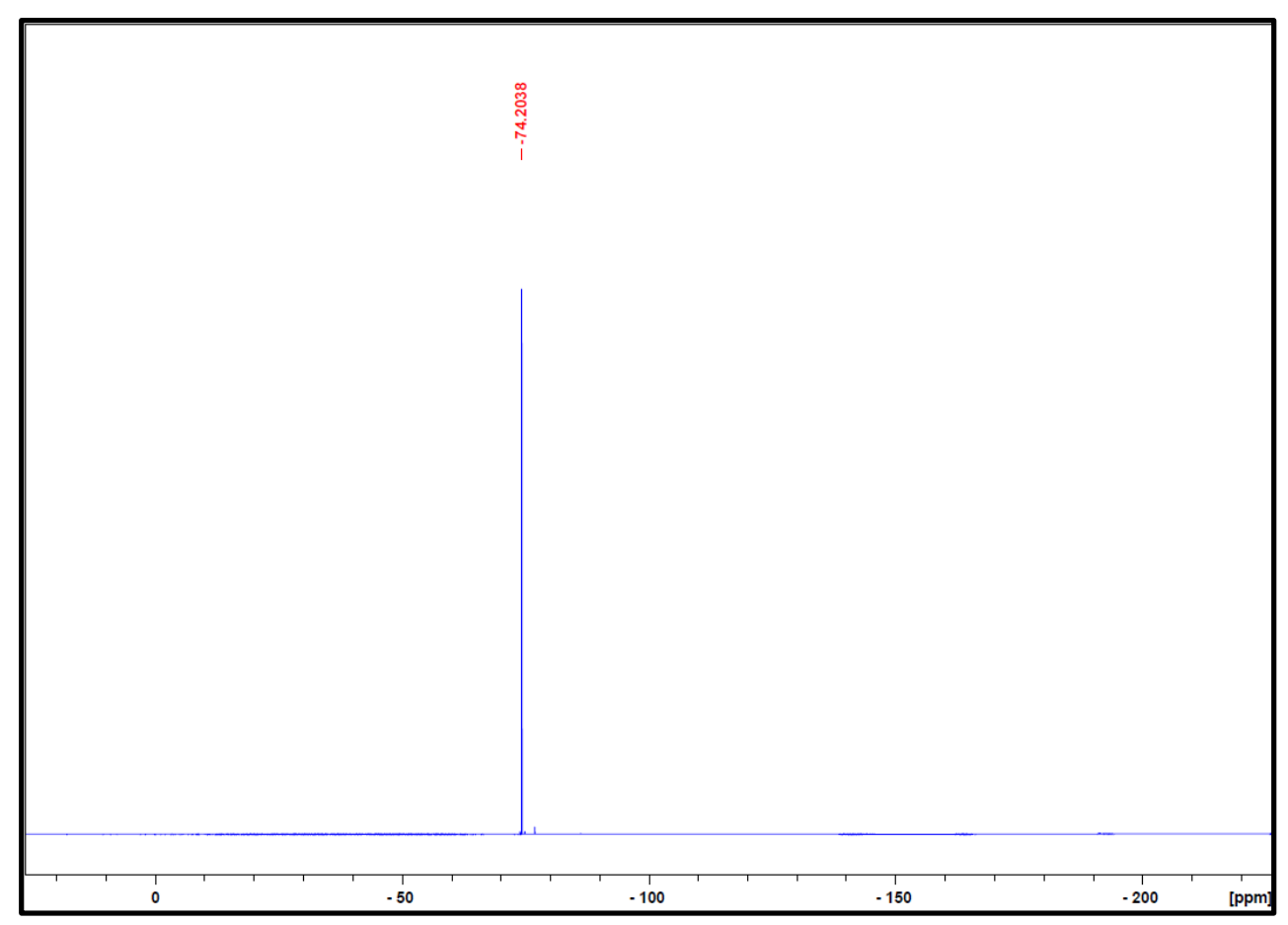

Figure S5: ${ }^{19} \mathrm{~F}$ NMR spectrum of $\mathbf{1}$, measured in $\mathrm{CDCl}_{3}$ at room temperature.

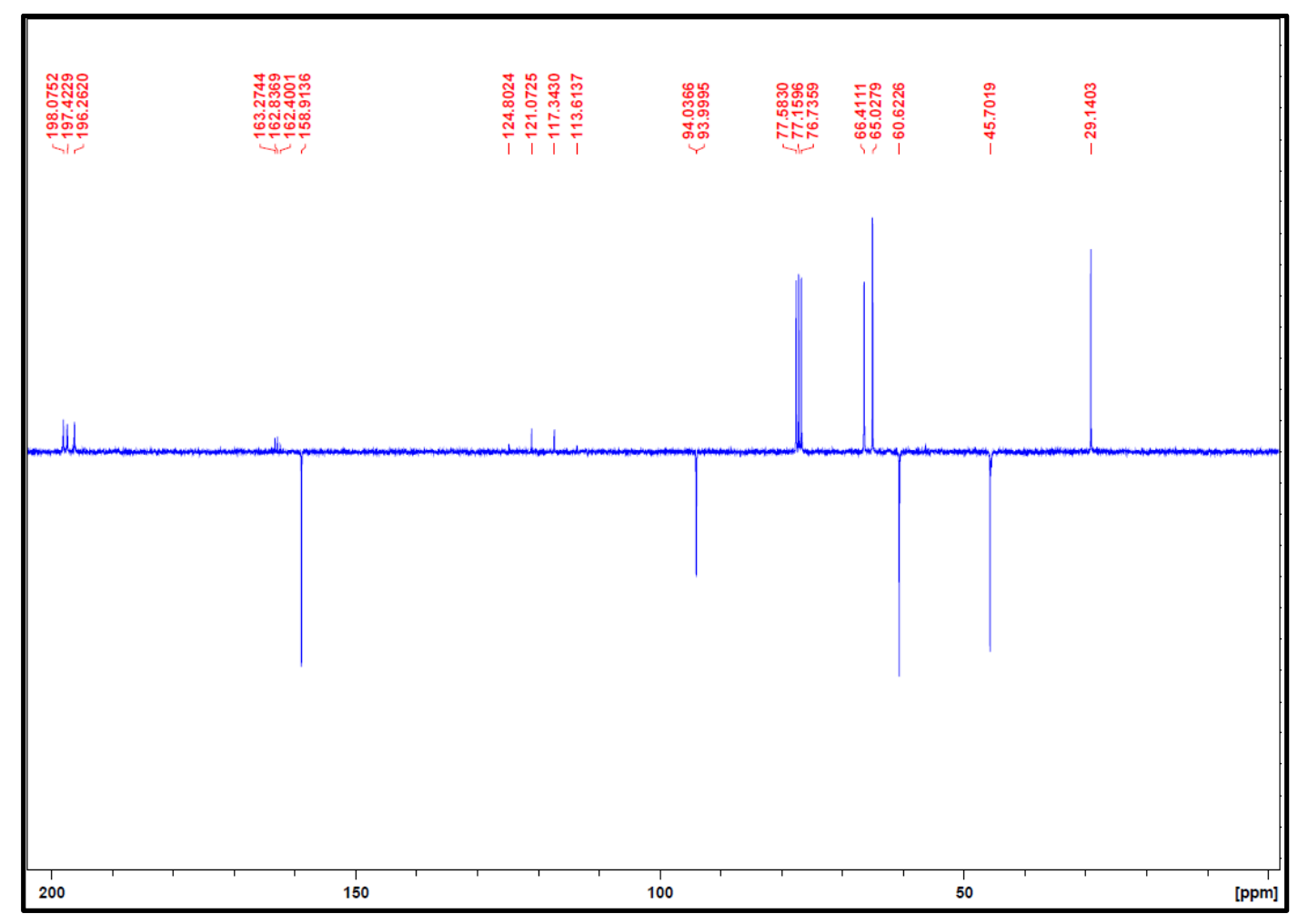

Figure S6: ${ }^{13} \mathrm{C}$ NMR spectrum of $\mathbf{1}$, measured in $\mathrm{CDCl}_{3}$ at room temperature. 


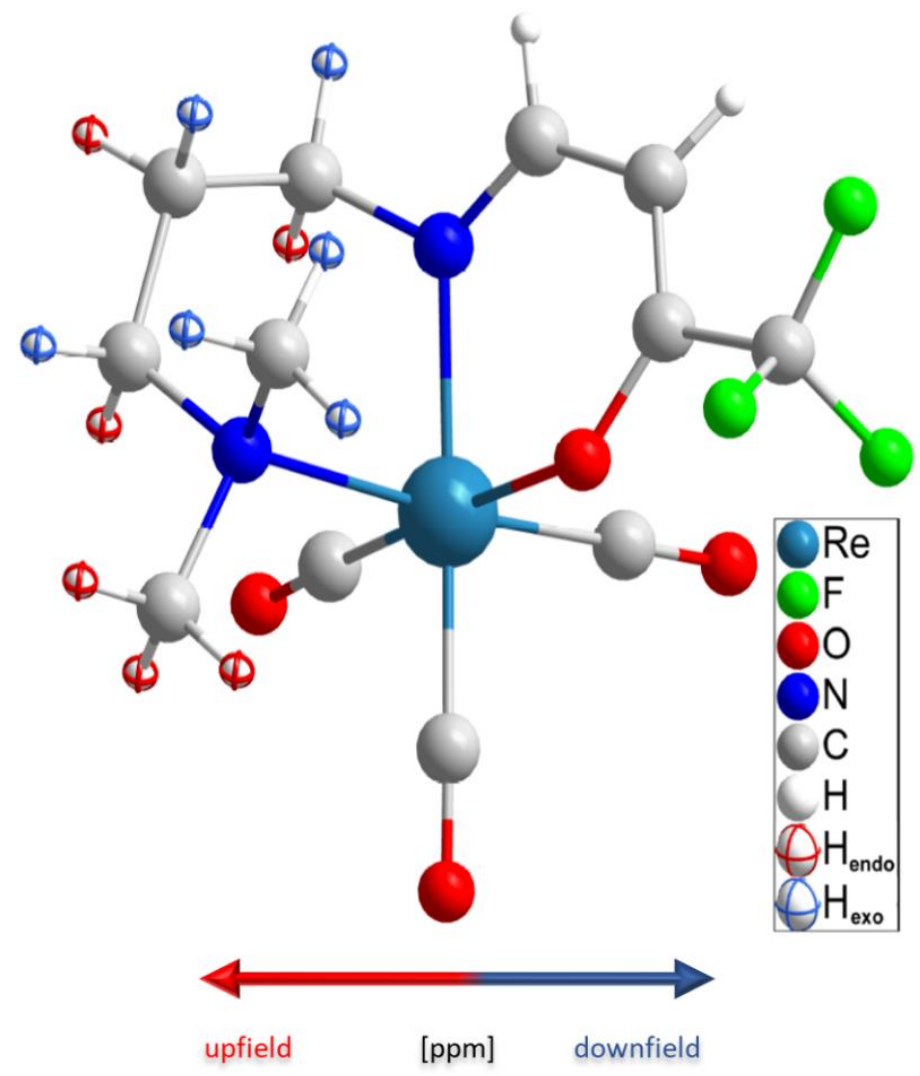

Figure S7: Schematic illustration of endo and exo protons in the saturated part of the tridentate ligand and the influence of their chemical shift in the NMR.

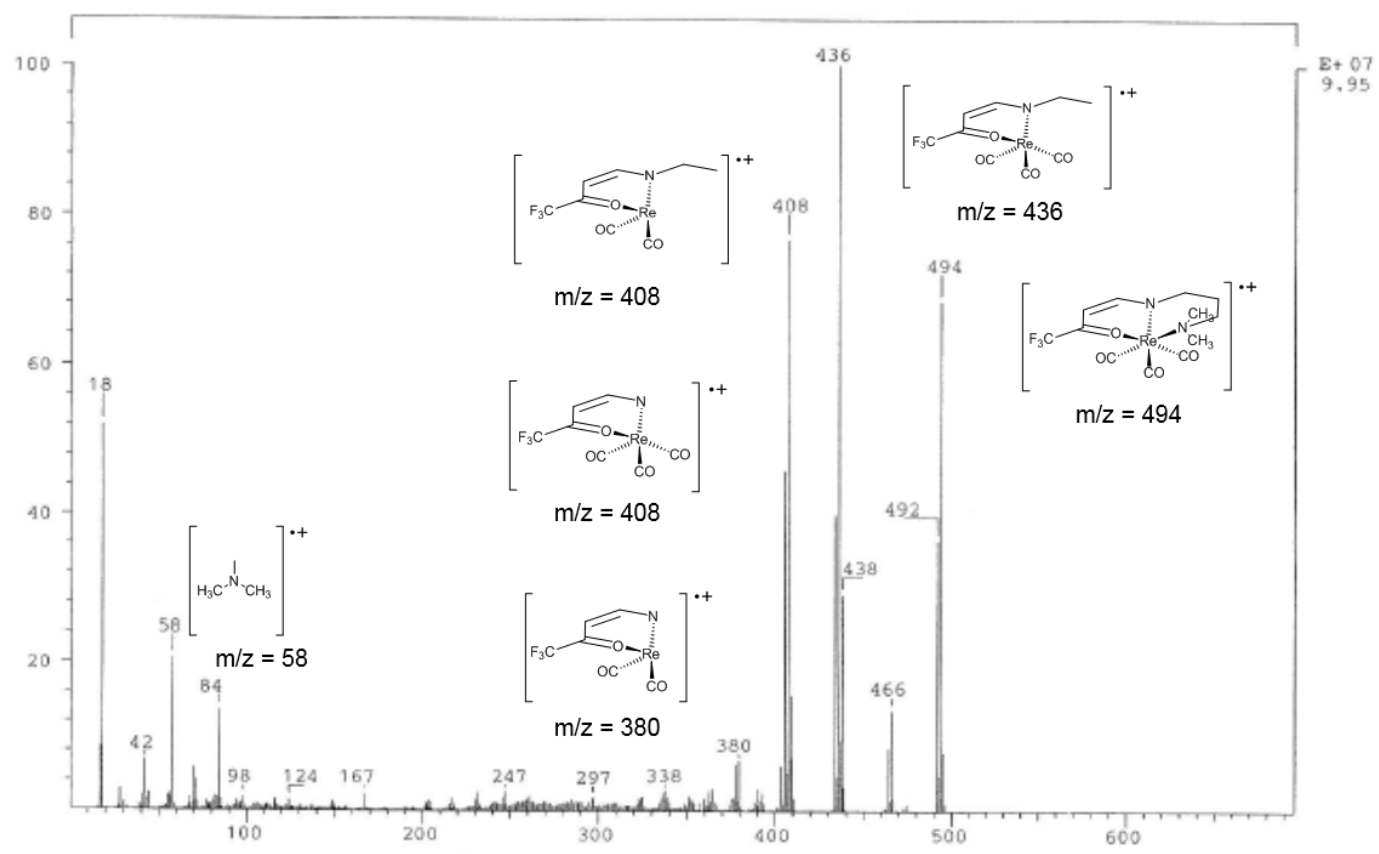

Figure S8: EI-MS spectrum of 1 at $70 \mathrm{eV}\left(10^{-6} \mathrm{mbar}\right)$. 


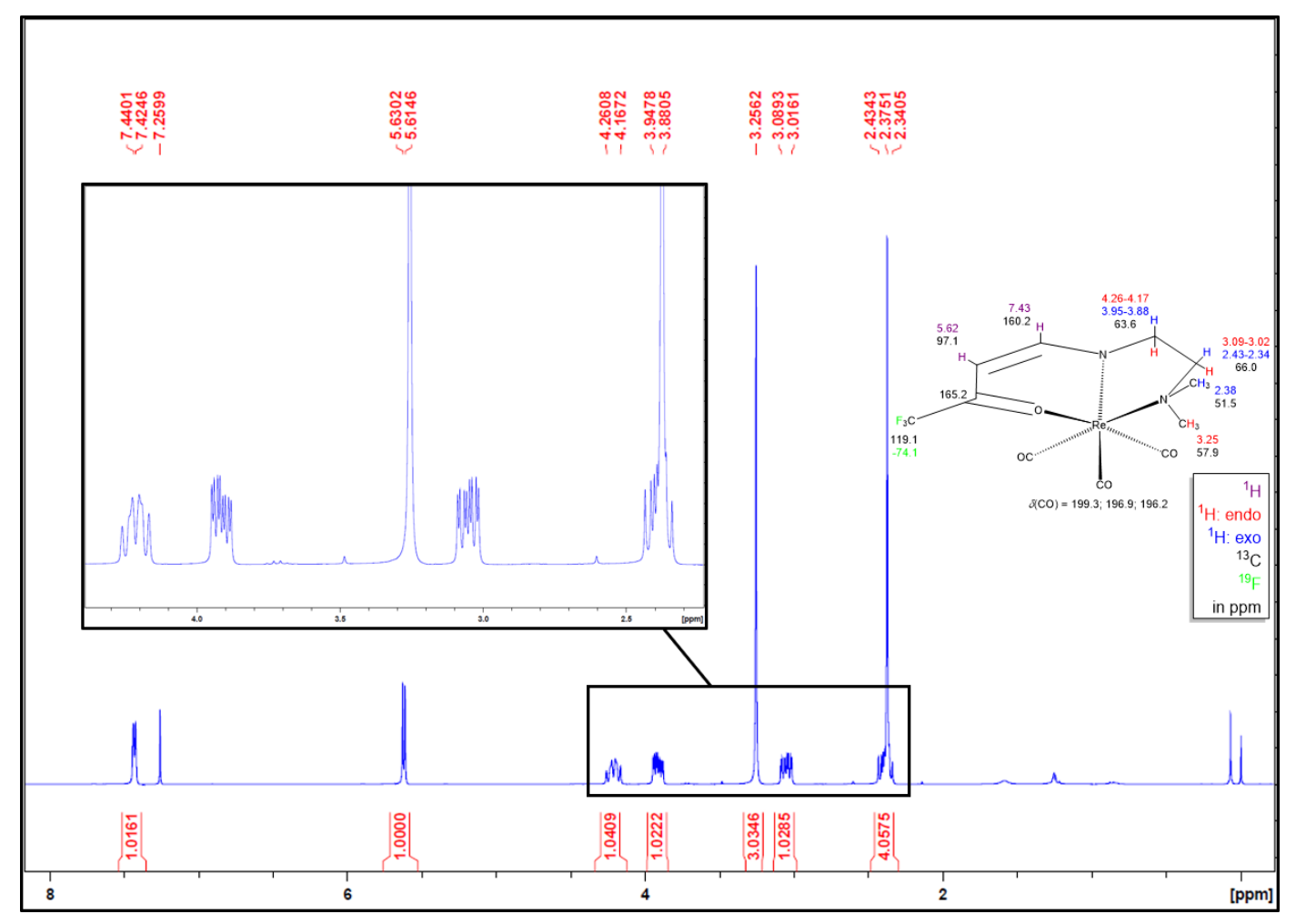

Figure S9: ${ }^{1} \mathrm{H}$ NMR spectrum of $\mathbf{2}$, measured in $\mathrm{CDCl}_{3}$ at room temperature.

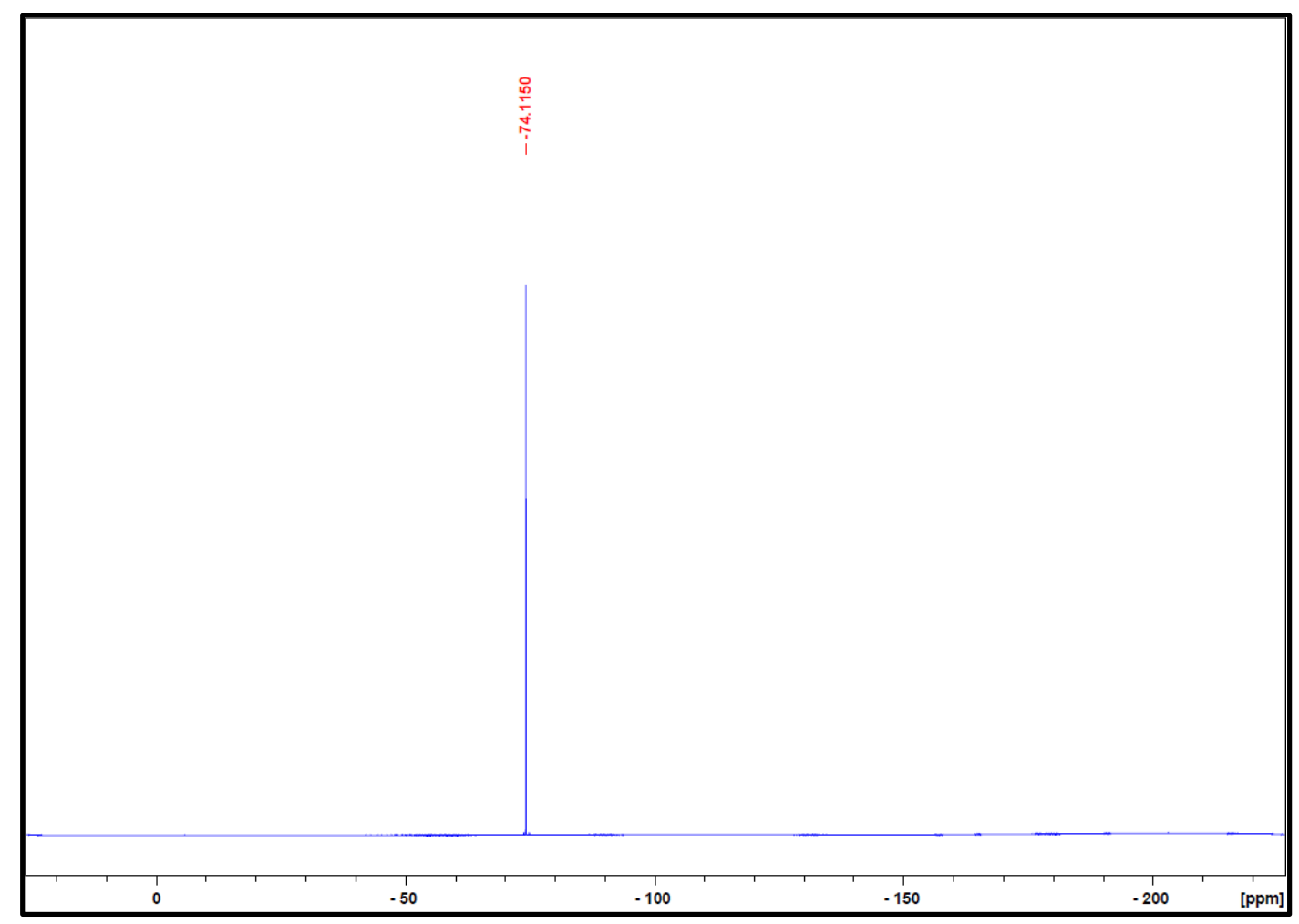

Figure S10: ${ }^{19} \mathrm{~F}$ NMR spectrum of 2 , measured in $\mathrm{CDCl}_{3}$ at room temperature. 


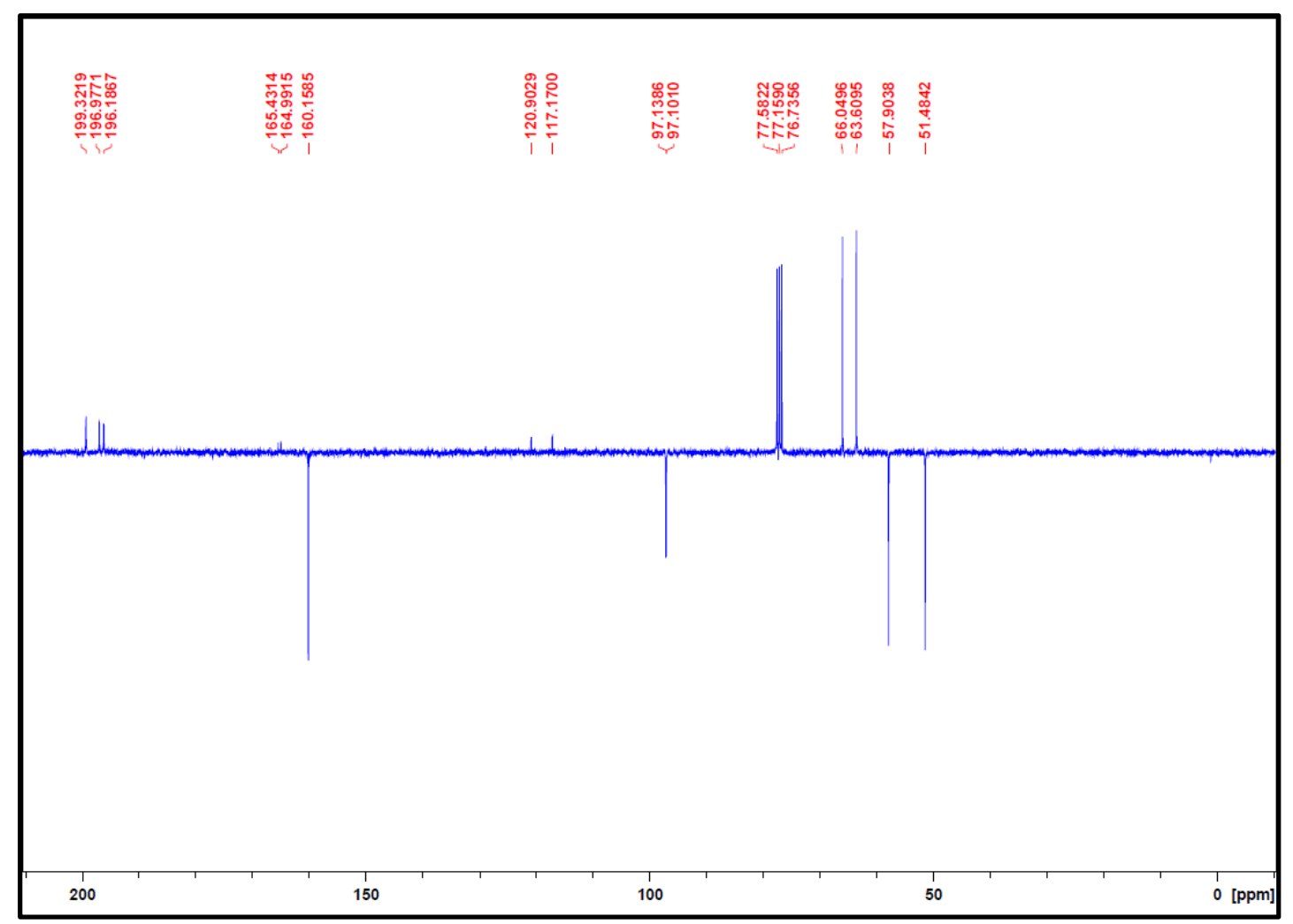

Figure $\mathrm{S} 11:{ }^{13} \mathrm{C}$ NMR spectrum of $\mathbf{2}$, measured in $\mathrm{CDCl}_{3}$ at room temperature.

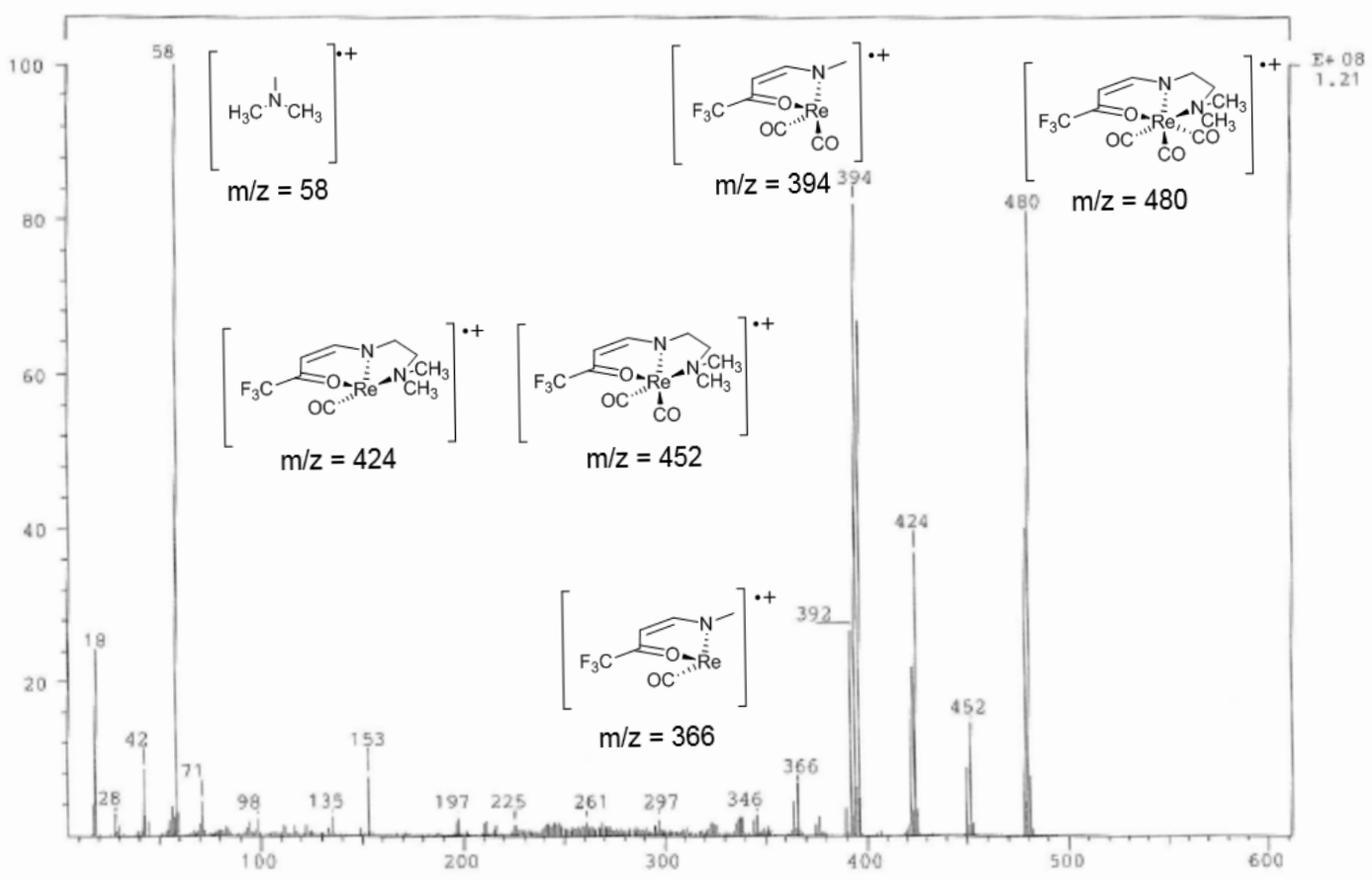

Figure S12: EI-MS spectrum of 2 at $70 \mathrm{eV}\left(10^{-6} \mathrm{mbar}\right)$. 


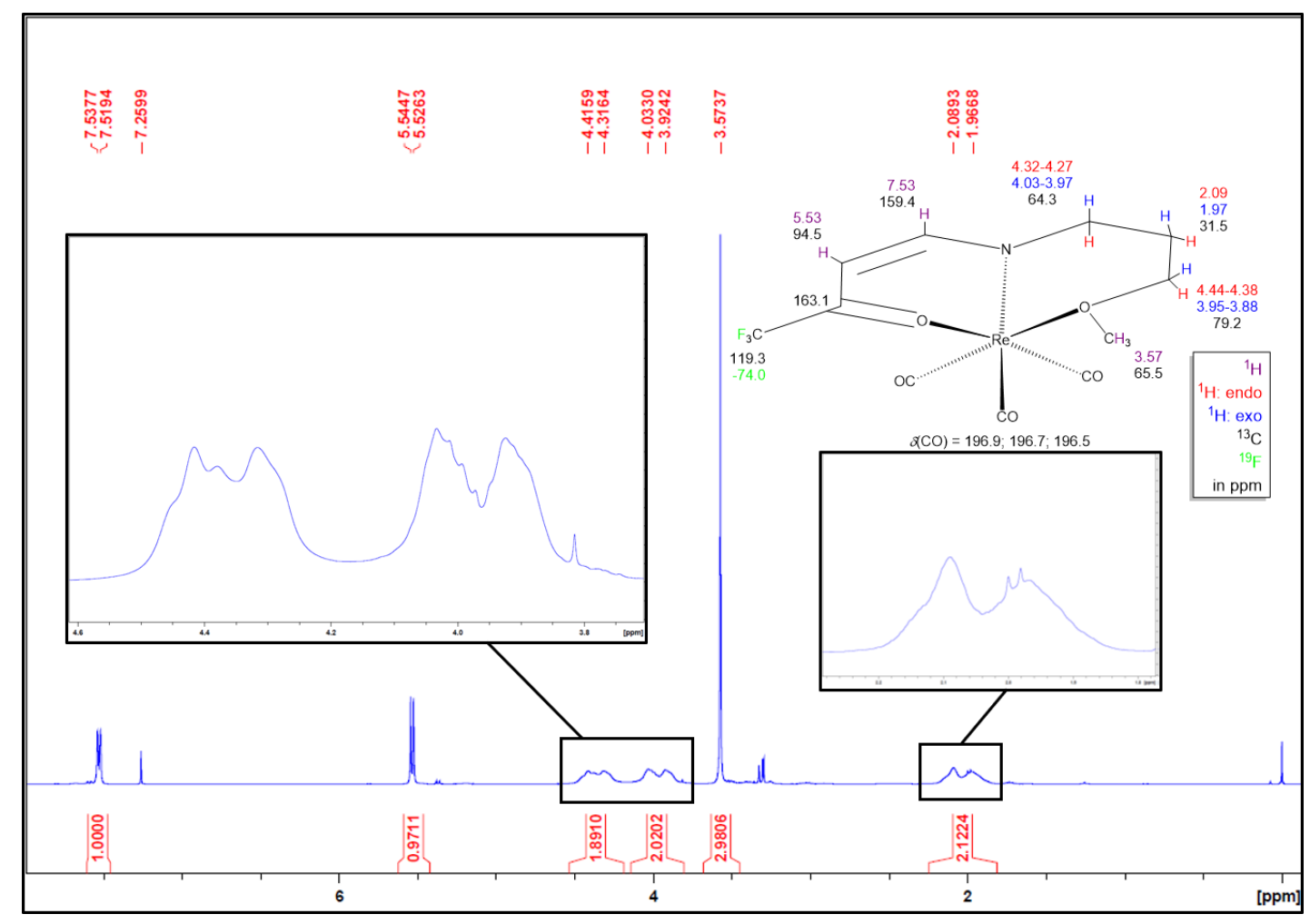

Figure S13: ${ }^{1} \mathrm{H}$ NMR spectrum of $\mathbf{3}$, measured in $\mathrm{CDCl}_{3}$ at room temperature.

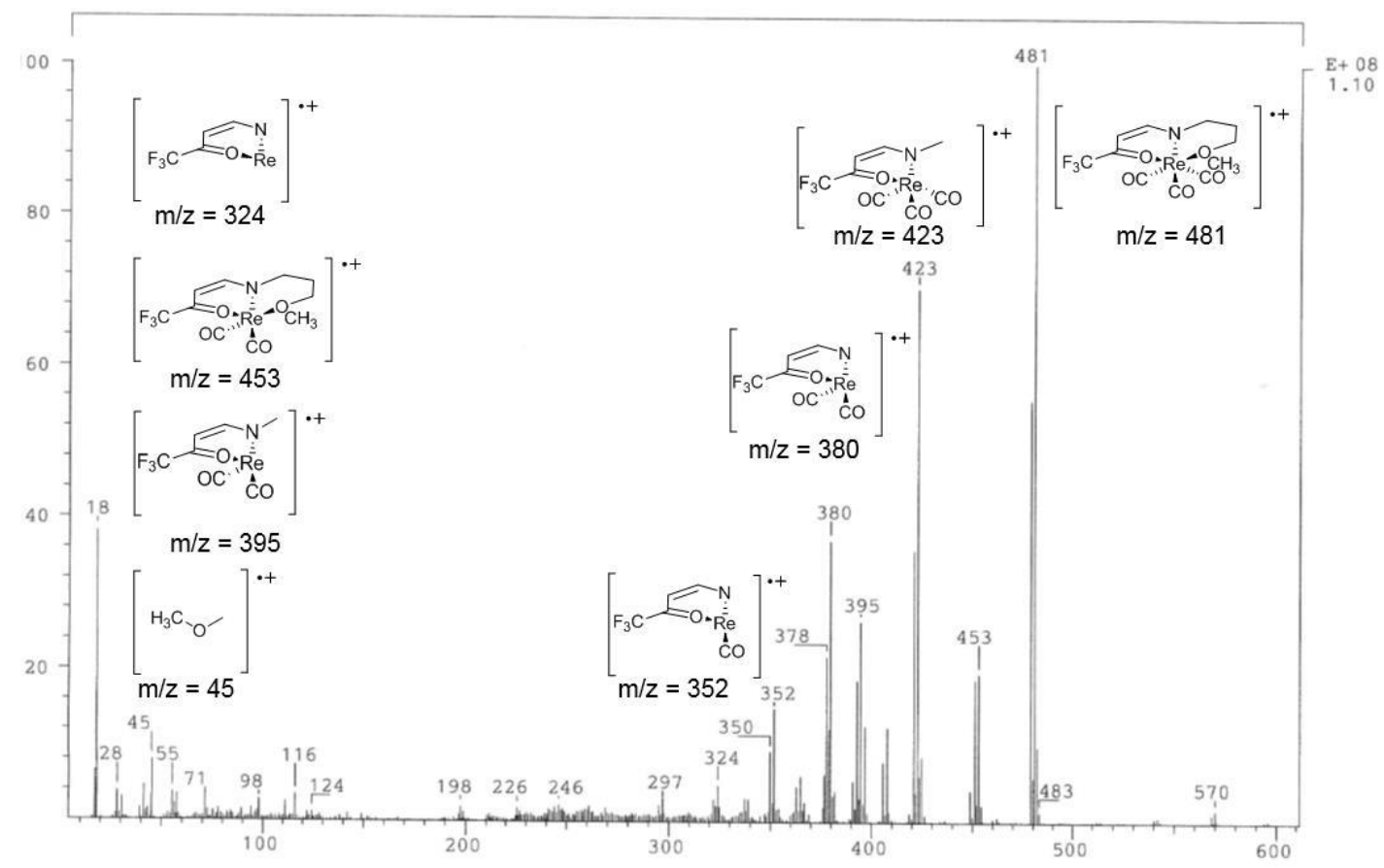

Figure S14: EI-MS spectrum of 3 at $70 \mathrm{eV}\left(10^{-6} \mathrm{mbar}\right)$. 


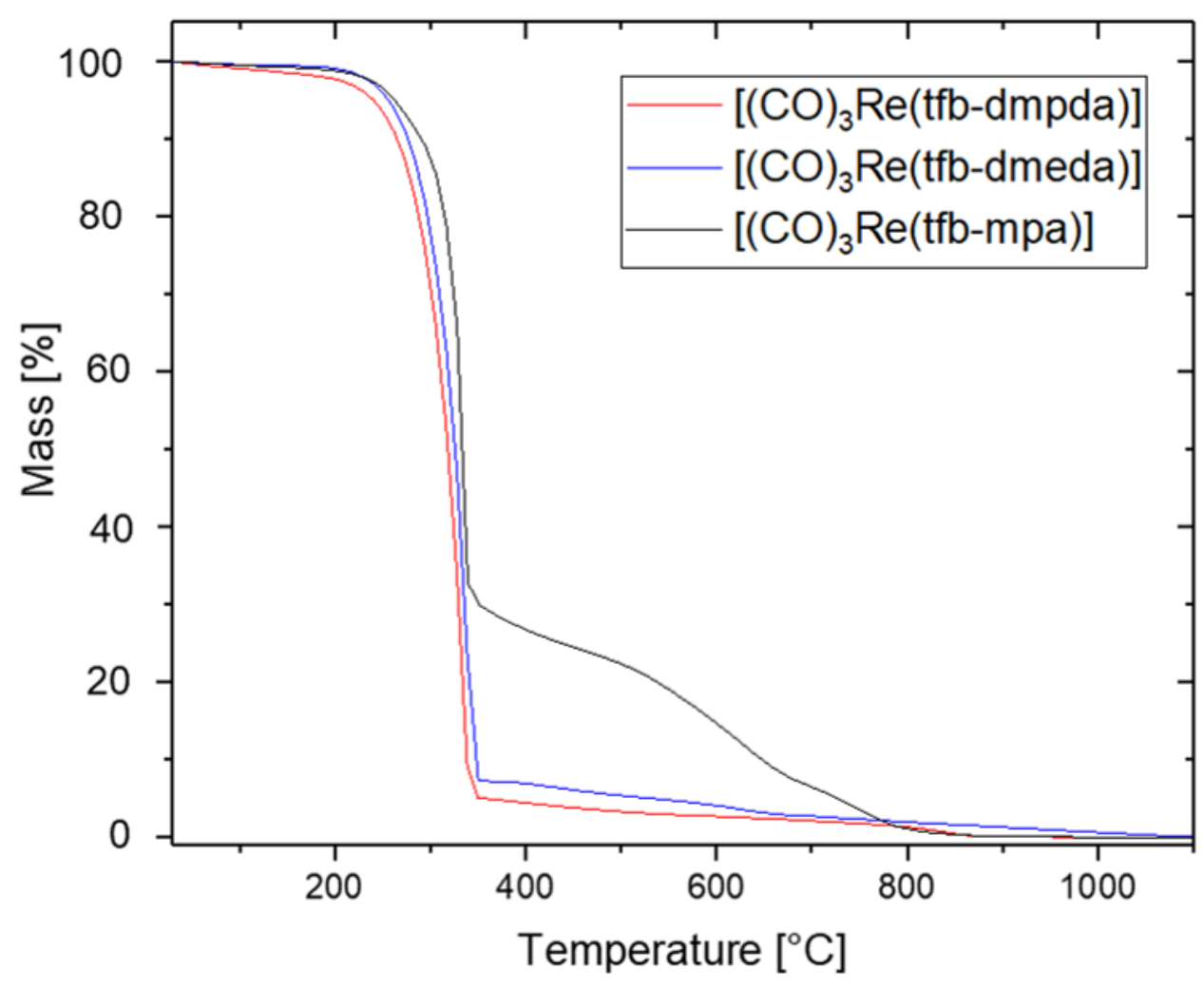

Figure S15: TG curves of $\mathbf{1 - 3}$, measured with a heating rate of $10{ }^{\circ} \mathrm{C} / \mathrm{min}$ under nitrogen.
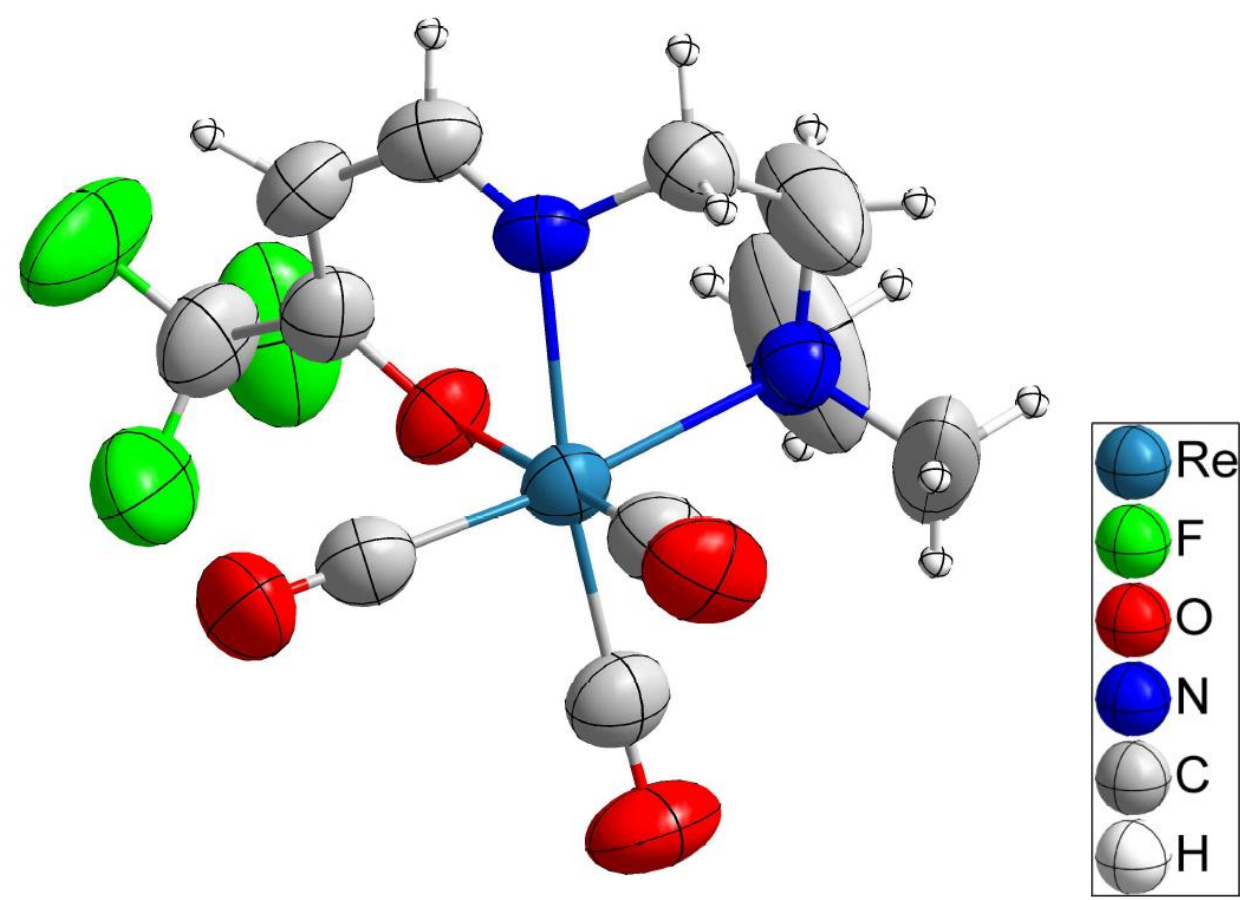

Figure S16: Molecular structure of 2. Thermal ellipsoids are drawn at 50\% probability level. 


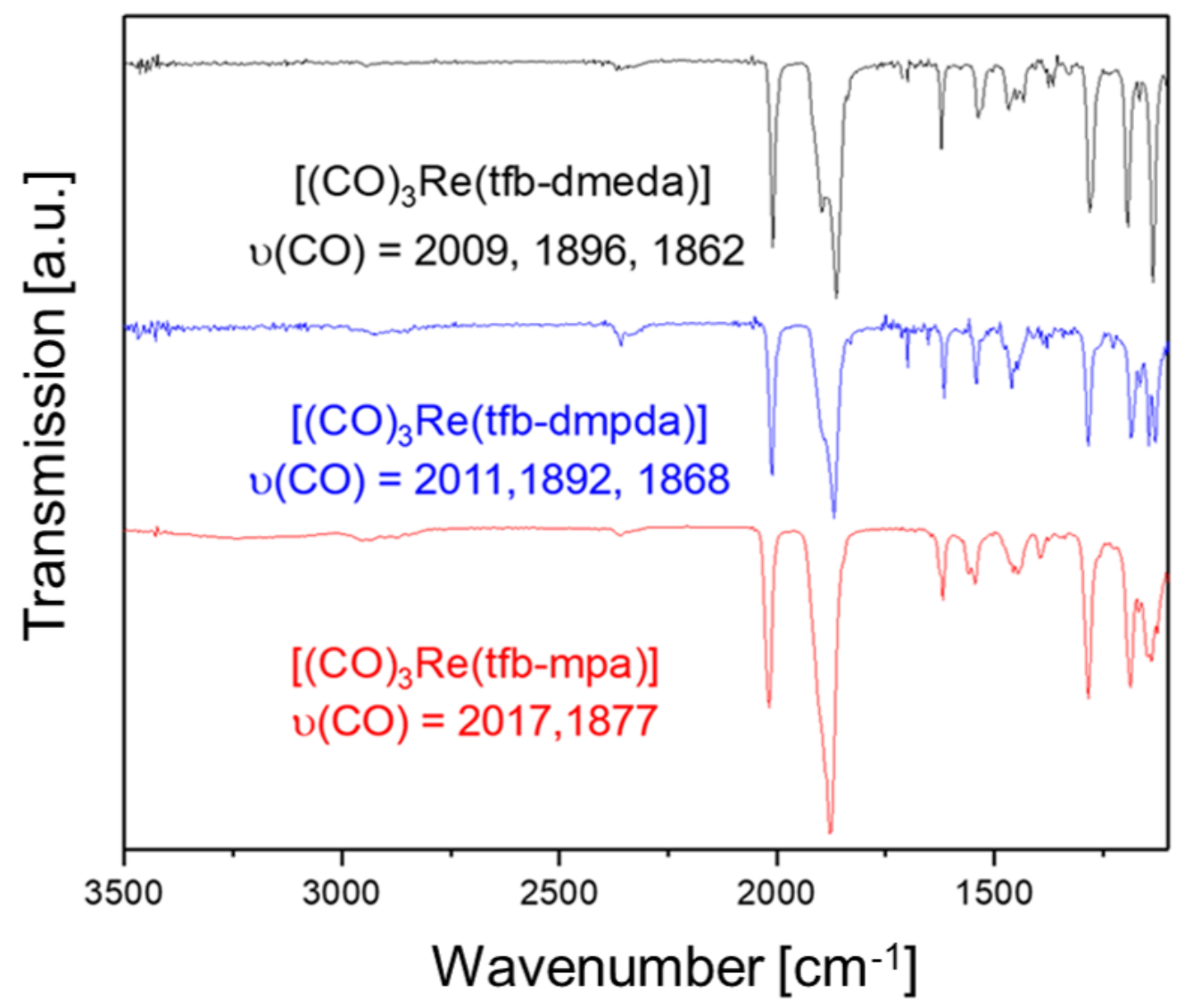

Figure S17: FT-IR spectra of 1-3.

Table S1: Crystal X-ray refinement data of Htfb-dmeda, Htfb-mpa, [(CO) $\left.{ }_{3} \operatorname{Re}(\mathrm{tfb}-\mathrm{dmpda})\right]$ and $\left[(\mathrm{CO})_{3} \operatorname{Re}(\mathrm{tfb}-\mathrm{dmeda})\right]$.

\begin{tabular}{|c|c|c|c|c|}
\hline & Htfb-dmeda & Htfb-mpa & $\begin{array}{l}{\left[(\mathrm{CO})_{3} \operatorname{Re}(\mathrm{tfb}-\mathrm{dmpd}\right.} \\
\text { a) }]\end{array}$ & $\begin{array}{l}{\left[(\mathrm{CO})_{3} \operatorname{Re}(\mathrm{tfb}-\mathrm{dm}\right.} \\
\text { eda) }\end{array}$ \\
\hline $\begin{array}{l}\text { Chemical } \\
\text { Formula }\end{array}$ & $\mathrm{C}_{8} \mathrm{H}_{14} \mathrm{~F}_{3} \mathrm{~N}_{2} \mathrm{O}$ & $\mathrm{C}_{8} \mathrm{H}_{12} \mathrm{~F}_{3} \mathrm{NO}_{2}$ & $\mathrm{C}_{12} \mathrm{H}_{14} \mathrm{~F}_{3} \mathrm{~N}_{2} \mathrm{O}_{4} \mathrm{Re}$ & $\mathrm{C}_{11} \mathrm{H}_{12} \mathrm{~F}_{3} \mathrm{~N}_{2} \mathrm{O}_{4} \mathrm{Re}$ \\
\hline Molar Mass & $211.21 \mathrm{~g} / \mathrm{mol}$ & $211.19 \mathrm{~g} / \mathrm{mol}$ & $493.45 \mathrm{~g} / \mathrm{mol}$ & $479.43 \mathrm{~g} / \mathrm{mol}$ \\
\hline $\begin{array}{l}\text { Temperature } \\
\text { of } \\
\text { Measurement }\end{array}$ & $170(2) \mathrm{K}$ & $170(2) \mathrm{K}$ & $170(2) \mathrm{K}$ & $293(2) \mathrm{K}$ \\
\hline $\begin{array}{l}\text { Crystal } \\
\text { System }\end{array}$ & Orthorhombic & Triclinic & Triclinic & Monoclinic \\
\hline Space Group & $P c c n$ & $P \overline{1}$ & $P \overline{1}$ & $P 21 / c$ \\
\hline $\begin{array}{l}\text { Lattice } \\
\text { Parameters }\end{array}$ & $\begin{array}{l}\mathrm{a}=15.2372(14) \AA \\
\mathrm{b}=10.7057(8) \AA \\
\mathrm{c}=12.3246(11) \AA \\
\alpha=90.00^{\circ} \\
\beta=90.00^{\circ} \\
\gamma=90.00^{\circ}\end{array}$ & $\begin{array}{l}\mathrm{a}=7.7204(6) \AA \\
\mathrm{b}=8.2960(7) \AA \\
\mathrm{c}=8.7882(7) \AA \\
\alpha=76.368(6)^{\circ} \\
\beta=69.590(6)^{\circ} \\
\gamma=77.153(7)^{\circ}\end{array}$ & $\begin{array}{l}a=7.3735(5) \AA \\
b=8.6840(6) \AA \\
c=12.5863(9) \AA \\
\alpha=78.378(6)^{\circ} \\
\beta=75.282(6)^{\circ} \\
\gamma=77.107(6)^{\circ}\end{array}$ & $\begin{array}{l}7.3108(3) \AA \\
15.2695(6) \AA \\
13.5491(7) \AA \\
\alpha=90^{\circ} \\
\beta=103.303(4)^{\circ} \\
\gamma=90^{\circ}\end{array}$ \\
\hline Cell Volume & $2010.4(3) \AA^{3}$ & $506.57(7) \AA^{3}$ & $750.81(10) \AA^{3}$ & $1471.93(12) \AA^{3}$ \\
\hline Formula unit & 8 & 2 & 2 & 4 \\
\hline $\begin{array}{l}\text { Goodness-of- } \\
\text { fit }\end{array}$ & 0.761 & 1.029 & 1.056 & 1.071 \\
\hline $\begin{array}{l}R_{1}\left[I_{0}>2 \sigma\right. \\
(I)] ; \text { all data }\end{array}$ & $0.0625 ; 0.1966$ & $0.0509 ; 0.0832$ & $0.0341 ; 0.0441$ & $0.0388 ; 0.0584$ \\
\hline
\end{tabular}




\begin{tabular}{|l|l|l|l|l|}
\hline $\begin{array}{l}\mathbf{w R} \mathbf{R}_{2}\left[\mathbf{I}_{\mathbf{0}}>\mathbf{2 \sigma}\right. \\
(\mathbf{I})] ; \text { all data }\end{array}$ & $0.1490 ; 0.2004$ & $0.1349 ; 0.1561$ & $0.0785 ; 0.0840$ & $0.0964 ; 0.1069$ \\
\hline
\end{tabular}
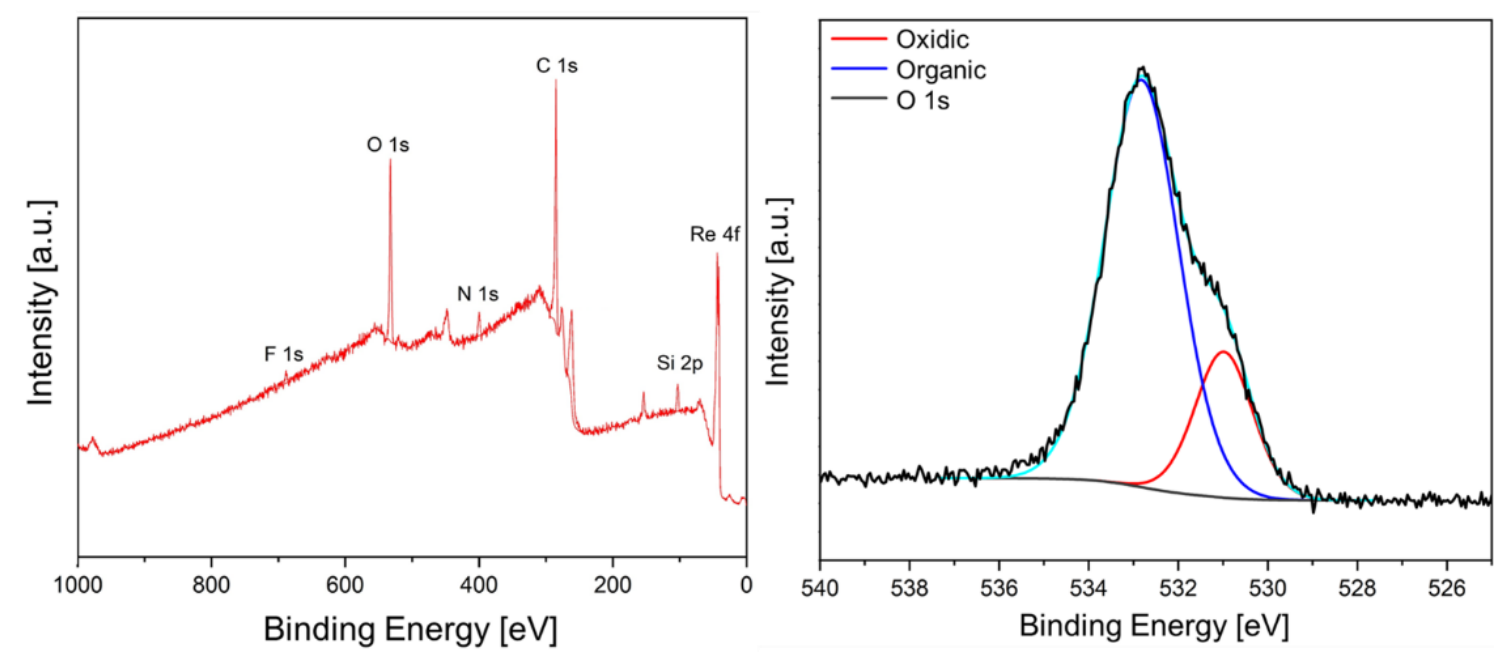

Figure S18: XPS survey spectrum (left) and high resolution O 1s spectrum (right) of the Rebased film deposited by MO-CVD at $600{ }^{\circ} \mathrm{C}$ on $\mathrm{Si}$.
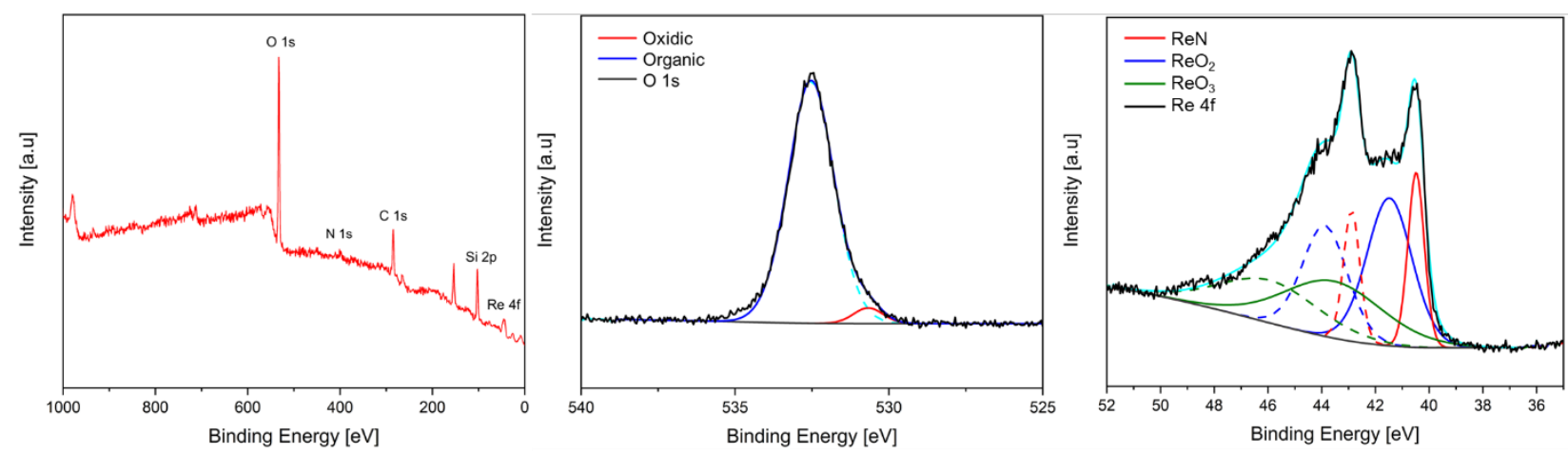

Figure S19: XPS survey spectrum (left), high resolution O 1s spectrum (middle) and high resolution $\mathrm{Re} 4 \mathrm{f}$ spectrum (right) of the Re-based film deposited by mfCVD at $600{ }^{\circ} \mathrm{C}$ on $\mathrm{Si}$.

Table S2: Elemental composition of the deposited films by MO-CVD and mfCVD on Si at $600{ }^{\circ} \mathrm{C}$ analyzed with XPS measurements.

\begin{tabular}{|c|c|c|}
\hline Element & MO-CVD & mfCVD \\
\hline Rhenium & $4.82 \%$ & $1.18 \%$ \\
\hline Nitrogen & $3.48 \%$ & $1.01 \%$ \\
\hline Carbon & $63.77 \%$ & $28.93 \%$ \\
\hline Oxygen & $19.79 \%$ & $36.54 \%$ \\
\hline Fluorine & $0.95 \%$ & $0 \%$ \\
\hline Silicon & $7.18 \%$ & $32.35 \%$ \\
\hline
\end{tabular}



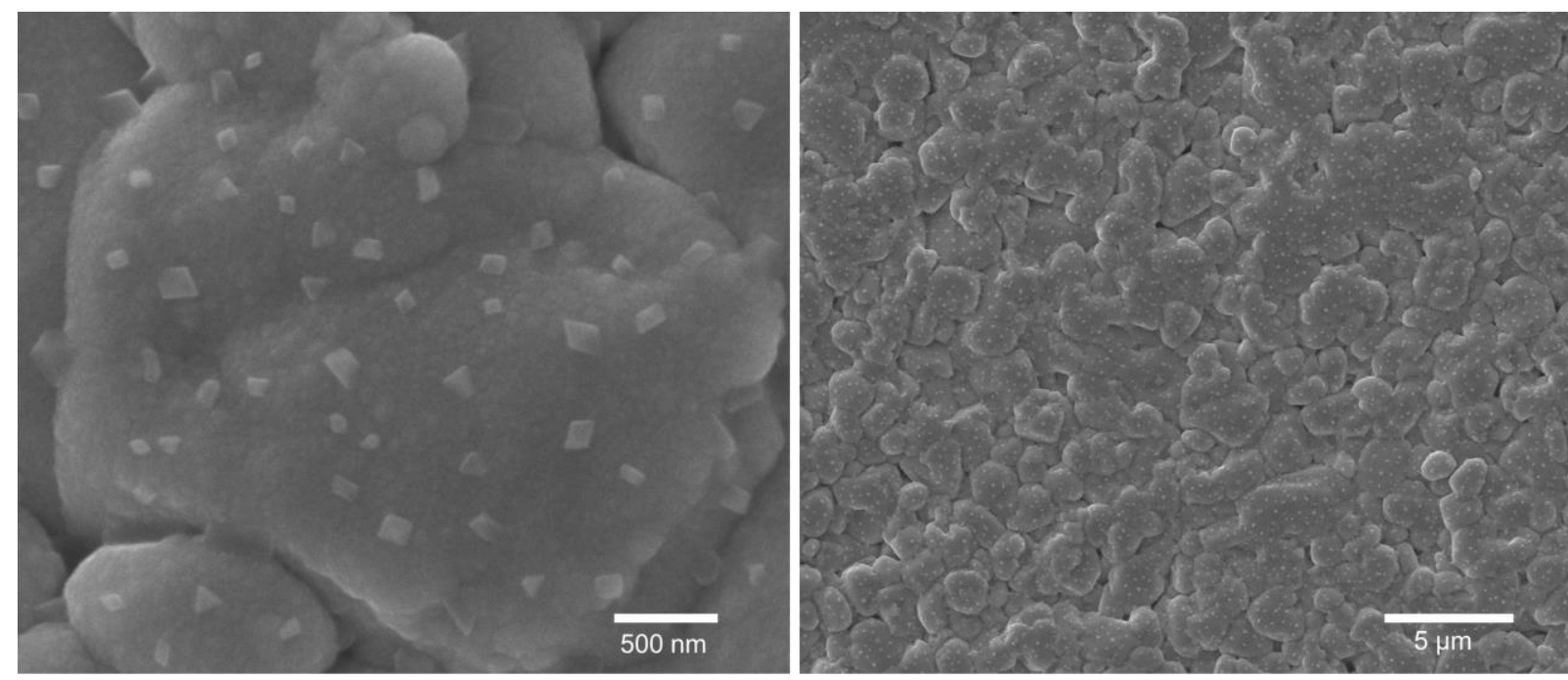

Figure S20: Top-view SEM images: Homogenous distributed ReN nanoparticles deposited via CVD on alumina substrate.

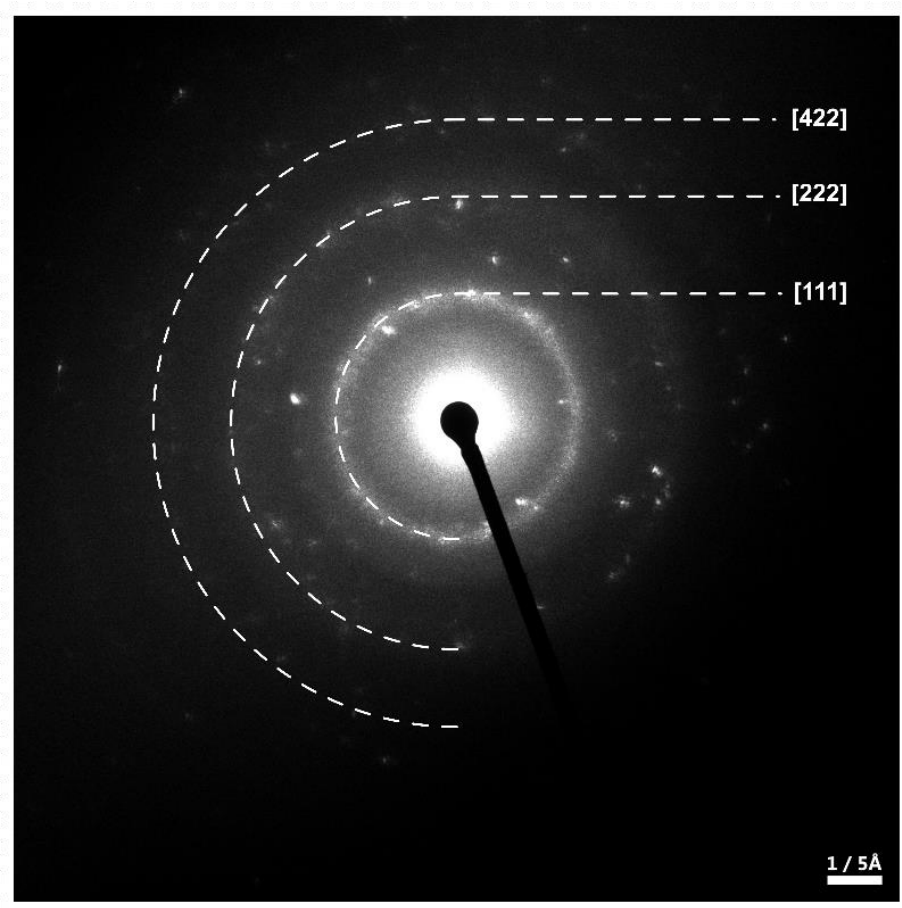

Figure S21: SAED of as deposited ReN (camera length: $600 \mathrm{~mm}$ ) transferred on $\mathrm{Cu}$ grids, indicating the [111], [222] and [422] planes (ICSD: 181297) of polycrystalline deposits. The preferred orientation visible on Si-substrates is lost due to the mechanical transfer to TEM sample holders. 